\title{
Association of Vitamin D with Type 2 Diabetes in Postmenopausal Females in Saudi Arabia
}

\author{
Shatha Alharazy ${ }^{1}$, Eman Alissa ${ }^{2}$, Mohammed Ardawi $^{2}$, Susan Lanham-New ${ }^{3}$ and \\ M. Denise Robertson ${ }^{3}$ \\ ${ }^{1}$ Department of Physiology, Faculty of Medicine, King Abdulaziz University, Jeddah, Saudi Arabia, \\ ${ }^{2}$ Department of Clinical Biochemistry, Faculty of Medicine, King Abdulaziz University, Jeddah, Saudi Arabia and \\ ${ }^{3}$ Department of Nutritional Sciences, Faculty of Health and Medical Sciences, University of Surrey, Guilford, United \\ Kingdom
}

\begin{abstract}
Vitamin D (vitD) deficiency has been suspected as a risk factor for type 2 diabetes mellitus (T2DM). It has been reported that an inverse relationship exists between vitD status and risk of T2DM. The aim of this study was to investigate whether there is an association between vitD status and glycemic profile and other metabolic parameters among postmenopausal women with T2DM (living in Saudi Arabia). A cross-sectional study was conducted at King Fahad Medical Research Center, King Abdulaziz University, Jeddah, Saudi Arabia. One thirty six $(n=136)$ postmenopausal females (age $\geq 50$ years) living in Jeddah city, Saudi Arabia, with T2DM were randomly recruited in this study. Anthropometric measures, blood pressure readings and fasting blood samples were obtained fro all study participants. Several biochemical parameters were estimated in fasting serum samples including total 25 $(\mathrm{OH}) \mathrm{D}, \mathrm{HbA}_{1 \mathrm{c}}$, insulin, glucose, c-peptide and lipid profile. Surrogate markers for insulin resistance were calculated using Homeostasis Model Assessment for insulin resistance and beta cell activity (HOMA-IR, HOMA- $\beta$ ), Quantitative insulin sensitivity check index (QUICK-I) and McAuley's index. VitD deficiency was defined as serum total 25(OH)D level below $20 \mathrm{ng} / \mathrm{ml}$. The Mean $( \pm \mathrm{SD})$ serum levels of total $25(\mathrm{OH}) \mathrm{D}$ were $13.8 \pm 8.6 \mathrm{ng} / \mathrm{ml}$ with $79 \%$ of the study cohort being vitD deficient. Furthermore, serum total $25(\mathrm{OH}) \mathrm{D}$ levels were found to be inversely correlated with fasting insulin $(r=-0.24, p=0.029)$, HOMA-IR $(r=-0.24, p=0.03)$, and positively correlated with McAuley's index $(r=0.22, p=0.048)$ and QUICK-I $(r=0.25, p=0.024)$. In conclusion, vitD deficiency is highly prevalent among postmenopausal women with T2DM living in Jeddah, Saudi Arabia. VitD was found to be associated with insulin resistance. Whether vitD supplements are able to improve insulin sensitivity and other parameters in T2DM postmenopausal women should be further investigated.
\end{abstract}

\section{Conflict of Interest}

There is no conflict of interest 